\title{
PROCESOS DE ORIENTACIÓN PARA LA INSERCIÓN SOCIOLABORAL DE PERSONAS CON ESPECIALES DIFICULTADES DE ACCESO AL MERCADO DE TRABAJO. UN MODELO DE ORIENTACIÓN PARA LA INSERCIÓN.
}

\author{
GUIDANCE PROCESSES FOR SOCIAL AND LABOUR INTEGRATION OF \\ PERSONS WITH SPECIAL DIFFICULTIES TO THE WORK MARKET \\ ACCESS. A GUIDANCE MODEL FOR THE EMPLOYABILITY
}

\author{
Pedro Jurado de los Santos* y Patricia Olmos Rueda** \\ Universidad Autònoma de Barcelona
}

\begin{abstract}
RESUMEN
En este trabajo, abordaremos algunos de los aspectos relacionados con los procesos de orientación para la inserción sociolaboral del colectivo de personas con especiales dificultades de acceso al mercado de trabajo, concretamente del colectivo de personas que han sido diagnosticadas con inteligencia límite. Analizaremos las principales dificultades que nuestra población destinataria encuentra a la hora de acceder al mercado de trabajo, los objetivos que persiguen los procesos de orientación, el papel de las competencias en estos procesos; especialmente las competencias de empleabilidad y adaptabilidad; y el planteamiento aproximativo a un modelo de orientación para la inserción laboral a partir de la autopercepción de las competencias.
\end{abstract}

Palabras clave: Orientación, inserción laboral, competencia, inteligencia límite, autopercepción.

\begin{abstract}
In this paper, we are going to talk about some aspects related to the guidance processes for social and labour integration of persons with special difficulties to the work market access, focusing on the collective of persons who have been diagnosed with limit intelligence. We are going to analyze the major challenges that our study collective find to the work market access, the aims of the guidance processes, and the role of competences in this process, especially de rol of employability and adaptability competences. For that, we propose a guidance model for the employability of this collective, which contrasted with the skills required in terms of work contexts, will be enable the analysis and detection of needs that will guide the process of take decisions.

\footnotetext{
* Director Dep. Pedagogía Aplicada. Líneas de investigación: Atención a las necesidades específicas: desde la escuela al mundo del trabajo y a la participación social. Percepción y actitudes hacia la diversidad. Integración e inclusión educativa. Integración e inserción sociolaboral de las personas con discapacidad. Transición escuela-trabajo. La formación de los profesionales y la atención a la diversidad. Análisis de necesidades formativas, evaluación y desarrollo profesional. La formación de formadores. Email: pedro.jurado@uab.cat

** Dep. Pedagogía Aplicada. Tesis de Máster La Formación Interna en la Empresa. Desarrollo de un Plan Integral de Formación Interna (Junio 2004). Líneas de investigación: La empleabilidad y la adaptabilidad de los jóvenes con I.L. y sus procesos de integración laboral. Modelo global de formación para la inserción en la empresa.
} 
The self-perception competences will be a key element in this guidance model if we will obtain an active and responsability involvement and participation of persons with limit intelligence in their process of training and guidance for incorporation into the work market. When our collective are aware of their competences, they will make it possible to self-identify themselves as capable and competent individuals, and instead, they are aware of their needs. It is the starting point of the guidance processes for social and labour integration of persons with special difficulties to the work market access.

Key words: Guidance processes, social and labour integration, competences, limit intelligence, self-perception.

\section{Introducción}

En la sociedad actual nos encontramos con colectivos considerados vulnerables debido a las dificultades que presentan para acceder al mercado de trabajo ordinario. La dificultad en el acceso al mercado de trabajo es una condición de vulnerabilidad que conduce a estos colectivos a situaciones de debilidad e indefensión frente a las exigencias sociales de "normalidad" de determinados sectores sociales. La dimensión social de la vulnerabilidad queda vinculada a la exclusión social, entendiendo esta última como una situación de desajuste producida por la inadecuada relación entre el sujeto, y el medio o entorno en el que vive y se relaciona (familiar, escolar, laboral, social, comunitario). Esta situación de desajuste lleva a los grupos considerados vulnerables ha estar al margen tanto de los procesos de integración habituales (trabajo, familia, organizaciones), como de las acciones establecidas para hacer frente a los fracasos de integración (Silver, 1994). Un ejemplo lo tenemos en el grupo de personas con algún tipo de discapacidad en edad laboral, y especialmente con discapacidad intelectual. Este colectivo despierta mayor inseguridad, rechazo y desconfianza debido a los prejuicios derivados del desconocimiento de sus potencialidades, y a los inconvenientes asociados a la adaptación de los puestos de trabajo y entornos laborales. Conclusiones de algunos estudios como los realizados por IDFO (1999) sobre las dificultades de acceso a la formación e integración laboral de las personas con discapacidad, o por Collet (2002) sobre las personas con discapacidad intelectual y las necesidades de apoyos intermitentes, permiten inferir la existencia de este bajo nivel de inclusión sociolaboral.

Esta realidad nos obliga a plantearnos cuestiones tales como qué papel juegan algunas competencias clave como la empleabilidad y la adaptabilidad en los procesos de acceso y permanencia en el mercado de trabajo de estos grupos vulnerables, o a considerar procesos de orientación para su inserción laboral.

\section{Desarrollo}

Sobre la población objeto de atención

Aproximarnos a nuestra población destinataria desde una perspectiva ecológica, implica considerar la discapacidad como consecuencia de la interacción de la persona con un ambiente que no le proporciona el adecuado apoyo para reducir sus limitaciones funcionales. Conocer los mediadores y 
facilitadores existentes hace que la discapacidad sea evidente o casi inapreciable. La influencia y papel del medio en la manifestación de la discapacidad, así como la presencia o ausencia de mediadores, apoyos y/o facilitadores que permitan las adaptaciones pertinentes, hace que dos personas con igual limitación manifiesten la misma de una u otra manera en función del contexto en el que se encuentren. Esta concepción de la discapacidad ha tenido implicaciones significativas en la consideración del importante papel que juega la conducta adaptativa en la reducción de las limitaciones funcionales (Schalock, 1999), y nos conduce a pesar la discapacidad intelectual en función del medio en el que la persona se desenvuelve; modelo biopsicosocial de la OMS (CIF, 2001); así como considerar toda una serie de mediadores, apoyos y/o facilitadores que permitan las adaptaciones pertinentes; modelo de prestación de apoyos de la AAIDD (Luckasson, 2002).

Desde esta perspectiva ecológica, el DSM-IV-TR (2001) propone el concepto de inteligencia límite (IL) cuando hay déficits en la conducta adaptativa asociados a una capacidad intelectual límite, estadísticamente relacionada entre 70 y 84 . A menudo, las personas con IL tienen dificultades para enfrentarse y adaptarse a las exigencias sociales y del entorno más inmediato y mediato. En general, el colectivo de personas con IL puede presentar dificultades en algunas habilidades de tipo social y de aprendizaje, pero son capaces de conseguir un alto grado de autonomía en las actividades de la vida diaria, con capacidad para autogobernarse y adaptarse. No obstante, si se apuesta por una integración con éxito, se tienen que conocer y considerar los aspectos que las hacen más vulnerables para poder ofrecerles los soportes más adecuados. Hay que decir, por otro lado, que la vulnerabilidad viene provocada por las situaciones sociales a las que hay que responder 0 adaptarse.

En definitiva, hablamos de un planteamiento sistemático y un trabajo cooperativo y colaborativo entre todos los agentes implicados en los procesos de orientación e integración sociolaboral; sujeto, familia, agentes sociales, profesionales de la orientación e integración, mundo empresarial y agentes políticos.

Dimensión social de la vulnerabilidad. Situaciones de desajuste desde la perspectiva de los procesos de orientación

La dimensión social de la vulnerabilidad queda vinculada a la exclusión social, entendiéndola como una situación de desajuste producida por la inadecuada relación entre el sujeto, y el medio o entorno en el que vive y se relaciona. Esta situación de desajuste lleva a los grupos considerados vulnerables ha estar al margen tanto de los procesos de integración habituales, como de las acciones establecidas para hacer frente a los fracasos de integración. Nuestro colectivo de estudio, las personas con IL, no quedan al margen de estas situaciones de desajuste y de exclusión sociolaboral. Ahora bien, ¿cómo definir la inadaptación sociolaboral desde la perspectiva de los procesos de orientación?.

Asumimos que las personas de referencia se encuentran en situación de desajuste en la relación entre una serie de capacidades afectivas, intelectuales, relacionales y laborales, y el nivel de funcionamiento exigido de esas 
capacidades por un contexto sociolaboral determinado. Esa situación de desajuste puede afectar al nivel de funcionamiento exigido de todas las capacidades, o bien sólo al nivel de funcionamiento de alguna de ellas, tal y como se expone en las situaciones que mostramos a continuación:

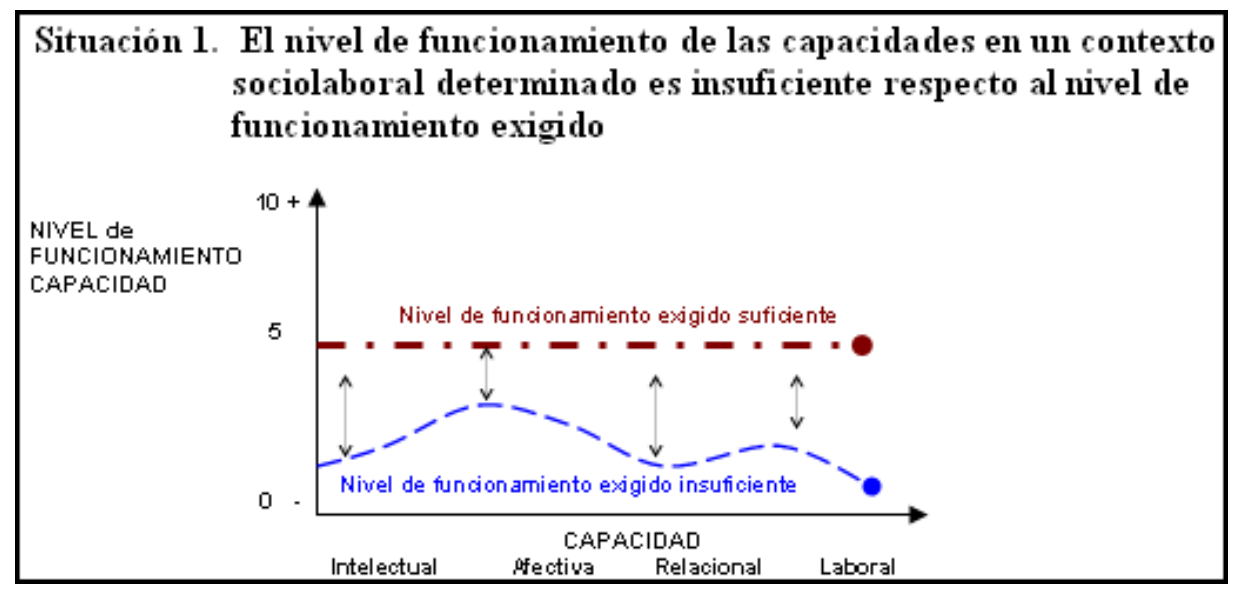

FIGURA 1.

Modelo de funcionamiento exigido de las capacidades insuficiente

En la situación planteada, existe una discrepancia entre la consideración de suficiencia en el funcionamiento de las capacidades analizadas y el nivel actual del sujeto, permitiéndonos avanzar los objetivos que debieran ponerse en marcha para disminuir dicha discrepancia, y posibilitar la adaptación al contexto específico a través de acciones de orientación.

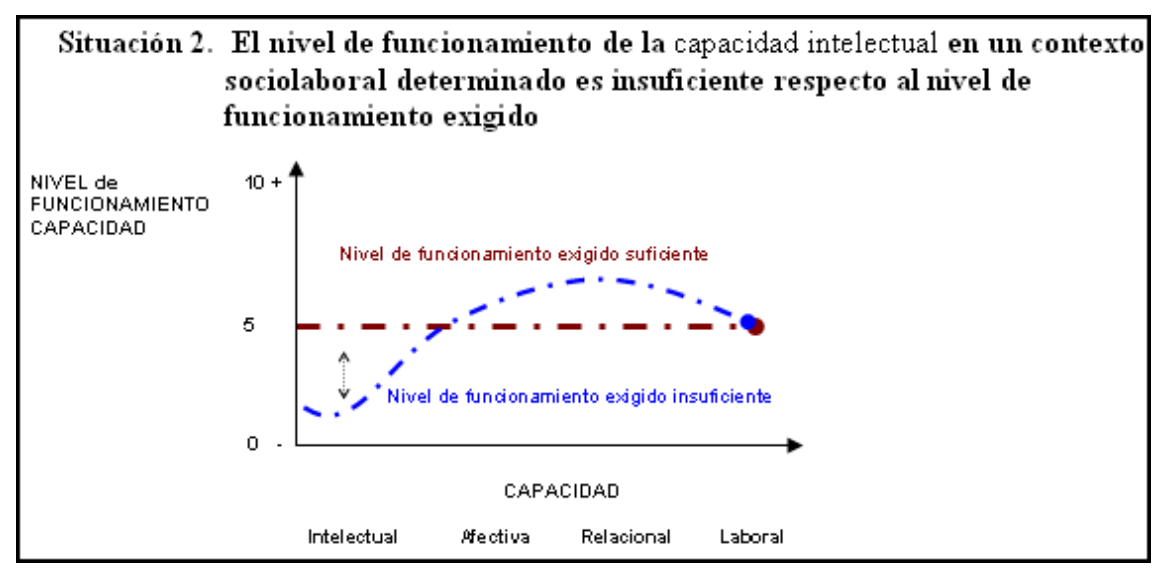

FIGURA 2.

Modelo de funcionamiento exigido de la capacidad intelectual insuficiente

En esta segunda situación, la discrepancia se da en la capacidad intelectual. Esta situación nos conduce a plantear procesos de orientación cuyo objetivo sea potenciar actividades que despierten el interés y la motivación hacia el aprendizaje, y que desarrollen y potencien la capacidad de aprender a aprender hasta conseguir el nivel de funcionamiento suficiente respecto a lo 
exigido por el contexto específico, como sucede con el resto de capacidades analizadas, posibilitando así la adaptación a dicho contexto.

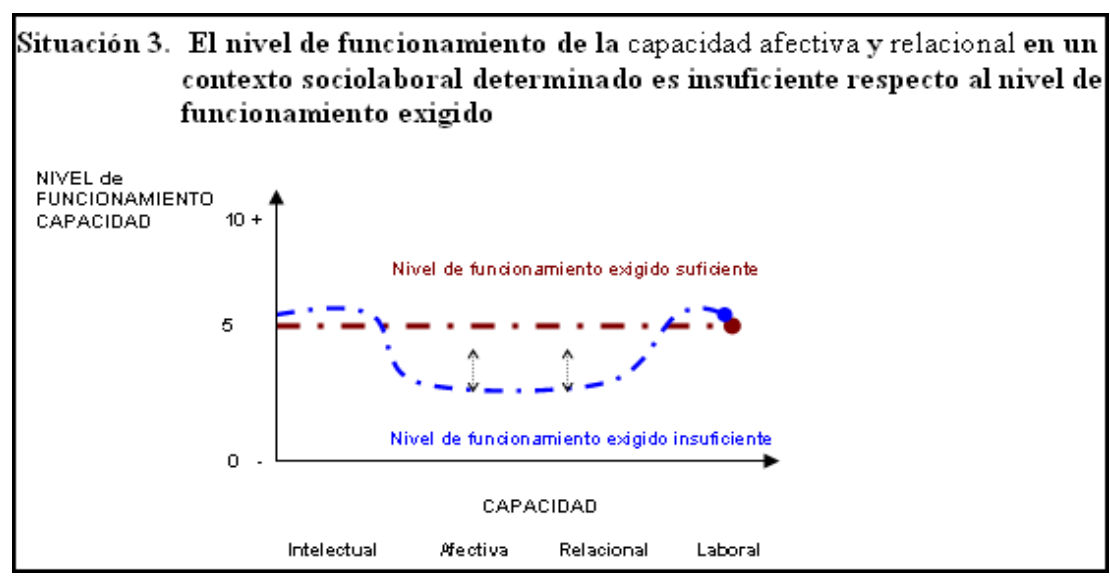

FIGURA 3.

Modelo de funcionamiento exigido de las capacidades afectiva y relacional insuficiente

Esta tercera situación plantea una discrepancia en las capacidades afectiva y relacional. En este caso, los procesos de orientación que se planteen deben potenciar la adquisición y desarrollo de competencias sociales y ciudadanas como son las habilidades sociales, la autonomía, la iniciativa, la capacidad de relación, entre otras, hasta conseguir que el nivel de funcionamiento sea suficiente respecto a lo exigido por el contexto específico posibilitando así la adaptación al mismo.

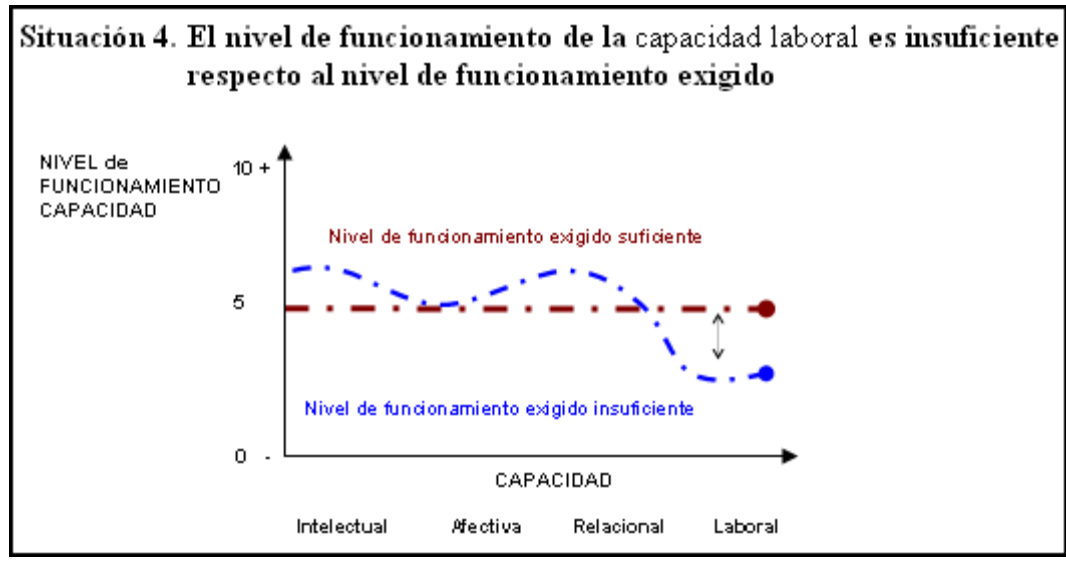

FIGURA 4.

Modelo de funcionamiento exigido de la capacidad laboral insuficiente

Una última situación nos platea la discrepancia en la capacidad laboral. Los procesos de orientación que se planteen tienen como objetivo conseguir un nivel de funcionamiento suficiente respecto a lo exigido por el contexto laboral específico potenciando y desarrollando competencias clave como son la empleabilidad y la adaptabilidad del sujeto a situaciones y/o contextos laborales 
dinámicos y cambiantes para así garantizar no sólo el acceso, sino también su permanencia y desarrollo profesional en los citados contextos.

Plantear un proceso de orientación sociolaboral exige partir del conocimiento del colectivo o población destinataria. No se puede emprender una actuación de orientación sin conocer qué define o caracteriza al colectivo de incidencia, ni tampoco obviar la relación existente entre la persona y el contexto. No obstante, tenemos que evitar caer en el error de asumir el colectivo como un individuo tipo, lo que lleva a determinar la necesidad de percibir en última instancia lo individual como clave del proceso de orientación.

Breves apuntes sobre la orientación para la inserción sociolaboral. el papel de la empleabilidad y la adaptabilidad

El INCANOP (Instituto Catalán de Nuevas Profesiones) define competencias básicas como el conjunto de elementos integrados por actitudes, aptitudes y conocimientos comunes en diferentes puestos de trabajo, oficios $o$ profesiones. En los contextos laborales son muchas las estrategias que se plantean respecto a la identificación y evaluación de las competencias, como la evaluación del puesto de trabajo que permite la detección e identificación de necesidades a partir del análisis de la ocupación en el lugar y en el entorno de trabajo. Asumir estos retos, nos lleva a identificar las necesidades en relación con las competencias y las exigencias del trabajo que deben realizar los trabajadores con discapacidad. En nuestro caso, planteamos la siguiente agrupación a partir de ocho competencias clave que son: Competencia matemática, en comunicación lingüística, en conocimientos e interacción con el entorno físico, en el tratamiento de la información y competencia digital, en el ámbito laboral, aprender a aprender, autonomía e iniciativa personal y competencia social y ciudadana. El grado de adquisición de estas competencias básicas va a influir en el éxito de la inserción sociolaboral.

Asumimos que los modelos, instrumentos o programas de orientación deben tener como objetivo la adquisición y desarrollo de las competencias referidas, que a su vez van a hacer emerger la empleabilidad y la adaptabilidad. Algunos autores (Fugate, 2004) definen la empleabilidad como un proceso proactivo de la adaptabilidad, y ambas constituyen competencias clave en los procesos de inserción laboral, y son determinantes en el éxito de acceso y permanencia al mundo del trabajo.

La orientación pretende aportar al individuo los instrumentos y herramientas necesarias para superar los obstáculos que pueden aparecer en su relación con el medio; ya sea laboral, educativo, social; tratando así de evitar o minimizar las consecuencias de una inadecuada adaptación entre una situación y otra generalmente nueva (Bermejo, 2007). Los procesos de orientación para el trabajo deben desarrollar las capacidades de adaptabilidad y empleabilidad de los sujetos en edad laboral; así, se incrementará la capacidad de dar respuesta a las exigencias del mercado de trabajo. Echeverría (1997) propone aplicar tres principios que deben guiar la acción orientadora en este ámbito: el principio de prevención, disponer el máximo de información contrastada; el de desarrollo, facilitar el tránsito hacia un estado de maduración; y el de intervención social, superar la dicotomía existente entre las expectativas y aspiraciones personales, y las exigencias del mundo laboral. 
Estos principios responden a las cuatro funciones principales del movimiento de educación para la carrera (Rodríguez Moreno, 1994): la conciencia de las oportunidades, la conciencia y conocimiento de sí mismo, la capacidad para la toma de decisiones y el aprendizaje para las transiciones.

En este proceso surgen toda una serie de programas, muchos de ellos iniciativas comunitarias, de promoción social del empleo y la formación, cuyo objetivo consiste en promover y mejorar las condiciones de acceso y permanencia al mercado laboral, asumiendo el principio de igualdad de oportunidades, así como mejorar la competitividad en el trabajo del colectivo de personas en edad laboral consideradas grupos de riesgo de exclusión sociolaboral. Algunos de estos programas se mencionan a continuación. HORIZON, trabaja con el colectivo de personas con discapacidad y su presencia en el mercado de trabajo; NOW y EQUAL con el colectivo de mujeres; YOUTHSTART con el colectivo de jóvenes sin cualificación; FORMA'T y PARTICIPA con el colectivo de jóvenes inmigrantes; INTEGRA agrupa al colectivo de personas con riesgo de exclusión del mercado de trabajo en general. Todos los referidos son programas en los que la orientación y formación juegan un papel prioritario en la adquisición de competencias profesionales, que van a preparar al colectivo de incidencia para su acceso y permanencia en el mercado de trabajo. Por lo tanto, deben plantearse acciones marcadas por la flexibilidad ente la orientación y la formación permanente, en la línea del lifelong learning, asumiendo la emergencia del concepto de empleabilidad (Kämäräinen, 2002).

Aproximación a un modelo de orientación para la inserción laboral de personas con inteligencia límite

Partimos del análisis de la autopercepción de las competencias básicas de las personas con IL para plantear un modelo de orientación cuyo propósito es orientar acciones que posibiliten la mejora de sus procesos de inserción en el mercado de trabajo ordinario.

A menudo, es en el momento de transición de la adolescencia y juventud hacia la vida adulta cuando se manifiesta la situación ligada al debate de "normal" y "especial" para estas personas. Esta transición debe estar ligada a la percepción de la situación de desventaja, la cual debe ser superada por parte del propio sujeto, dado que generalmente aporta una historia de fracaso que requiere de soporte para conseguir el objetivo de la inserción en el mundo del trabajo. Entendemos que la percepción sobre uno mismo se relacionará con el autoconcepto, de tal manera que podemos hipotetizar:

- un alto concepto de sí mismo promoverá en el sujeto acciones de mayor impulso hacia la superación de los problemas y avanzar hacia la autodeterminación, y

- un bajo concepto de sí mismo hará que la situación del sujeto sea proclive a establecer acciones de dependencia.

Es interesante apuntar que una propuesta de orientación que entienda las autopercepciones de los sujetos con relación a sus competencias será útil para proveer mecanismos que faciliten los procesos de inserción. En este sentido hemos de entender las competencias básicas como claves para 
responder a la adaptabilidad y promover la empleabilidad de los sujetos. Todas aquellas informaciones del sujeto además de plantear sus niveles competenciales pueden ser objeto de análisis a partir de su pensamiento con relación a su capacidad de respuesta. Para ello planteamos el siguiente modelo de orientación:

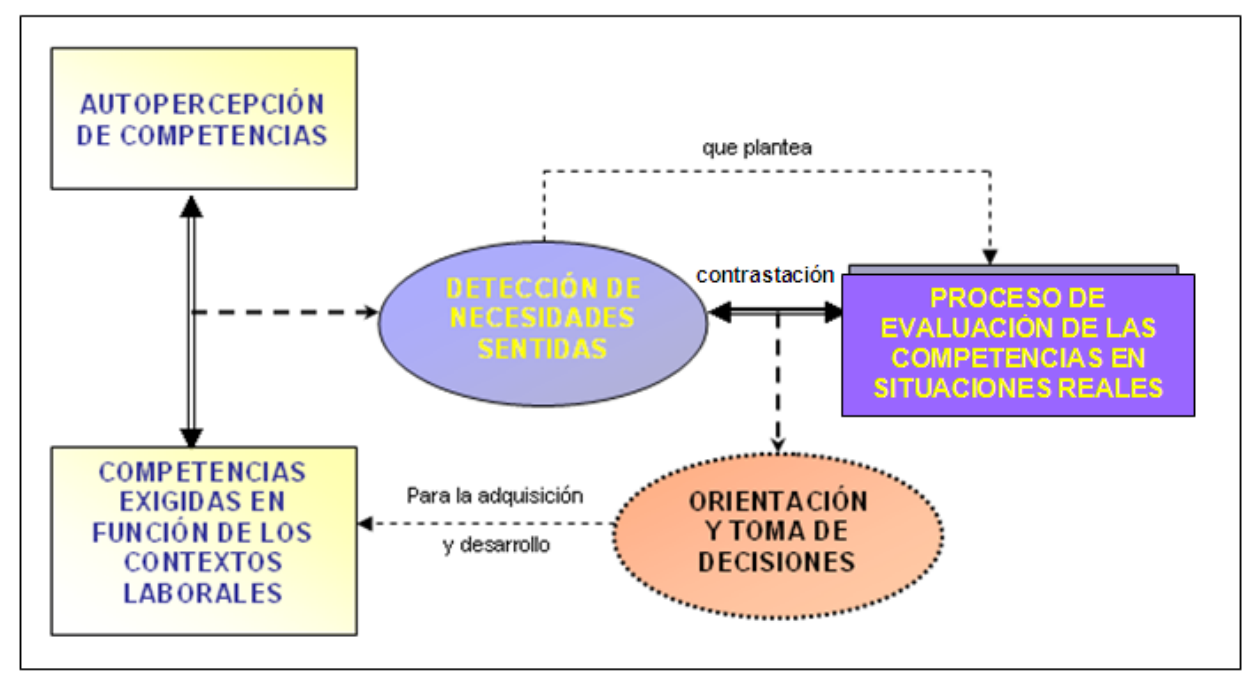

FIGURA 5.

Modelo de orientación para la inserción laboral de las personas con inteligencia límite

Partimos de la detección de necesidades sentidas por el sujeto a partir del análisis de discrepancias entre la autopercepción de las competencias por parte del sujeto, y las competencias laborales exigidas en función de los contextos de trabajo. A partir de esta detección de necesidades se planteará un proceso de evaluación de las competencias en situaciones reales, proceso que será contrastado con la detección de necesidades realizada y planteará acciones de orientación y toma de decisiones para la adquisición y desarrollo de las competencias laborales exigidas.

La autopercepción se asume como un factor que contribuye a considerar el concepto de sí mismo en torno a las posibilidades basadas en la identidad propia, en las creencias sobre sí mismo y, por tanto en las expectativas fundamentadas sobre el yo de los sujetos. El contraste con las exigencias exigidas o establecidas para el ejercicio profesional en un lugar de trabajo, permite establecer el nivel de discrepancias sobre las que se van a plantear las necesidades sentidas, como resultado de un factor de aproximación para orientar y tomar decisiones, relacionadas bien con procesos formativos o de incorporación al contexto laboral.

No obstante, habrá que tener en cuenta que un análisis exhaustivo de las competencias actuales del sujeto debe proveerse atendiendo a procedimientos tales como el de evaluación en el trabajo, además de considerar sus aprendizajes previos.

\section{Conclusiones}


A lo largo de este trabajo se ha planteado los procesos de orientación para la inserción laboral de las personas con especiales dificultades de acceso al mercado de trabajo, y concretamente de las personas con capacidad intelectual límite. Se apunta que el propósito de la orientación es conseguir que toda persona sea capaz de adquirir y desarrollar las competencias básicas necesarias para su incorporación y mantenimiento en el mercado de trabajo ordinario. Asimismo, no pueden olvidarse dos de los ejes clave de actuación para la consecución de dicho propósito: el desarrollo de la empleabilidad y de la adaptabilidad. Todo esto, nos permite avanzar un modelo para la orientación permanente y flexible a lo largo de la carrera socioprofesional de nuestro colectivo de estudio, que analice y valore las competencias clave relacionadas con el acceso y permanencia al mercado de trabajo de las personas con capacidad intelectual límite, y que oriente la propuesta de acciones para la adquisición y desarrollo de las competencias. El planteamiento de este modelo deberá partir de dos ejes básicos de actuación: (1) el conocimiento de las personas con capacidad intelectual límite, de sus potencialidades y de sus limitaciones, así como la influencia del contexto sociolaboral en el que se desarrollan atendiendo a las exigencias del mismo, y (2) el análisis de la autopercepción de las competencias básicas de las personas con capacidad intelectual límite en el momento de transición hacia la vida adulta dado que es el momento de mayor percepción de la situación de desventaja que va a requerir, por parte del sujeto, del soporte necesario para conseguir su inserción y permanencia en el mundo del trabajo.

\section{Referencias Bibliográficas}

BERMEJO, B. (2007). Orientación y desarrollo profesional. [Consulta: 15/10/2007] http://tecnologiaedu.us.es/formacionytrabajo/ponencias/blasbermejo.htm

COLLET, M. et alt. (2002). Personas con discapacidad intelectual y necesidades de apoyos intermitentes: situación, necesidades y demandas. Barcelona: IMSERSO.

COLLET, M. et alt. (2004). Aproximación sociológica a las personas con DINAI. MinusVal, (145), 13-15.

DSM-IV (2001). Manual Estadístico de desórdenes Mentales. Barcelona: Masson.

ECHEVERRÍA, B. (1997). Inserción sociolaboral. Revista de Investigación Educativa, 15(2), 85-115.

FUGATE, M., KINICHI, A.J. y ASHFORTH, B.E. (2004). Employability: A psycho-social construct, its dimensions and applications. Journal of Vocational Behaviour, (65), 14-38.

IDFO (1999). Nuevas oportunidades de integración social para las personas con discapacidad. Barcelona: IDFO-UGT.

LUCKASSON, R. y cols (2002). Mental Retardation: Definition, Classification, and Systems of Suports (10 ${ }^{\text {th }}$ Edition). Washington: AAMR. Versión española de M.A. VERDUGO, M. y C. JENARO (1997). Retraso mental: definición, clasificación y sistemas de apoyo / American Association on Mental Retardation. Madrid: Alianza Editorial. 
MARIÑO, F. y FERNÁNDEZ, C. (coords.) (2001). La protección de las personas y grupos vulnerables en el derecho europeo. Madrid: Ministerio de Trabajo y Asuntos Sociales.

ORGANIZACIÓN MUNDIAL DE LA SALUD (2001). Clasificación Internacional del Funcionamient, de la discapacidad y de la salud: CIF. Ginebra: OMS.

PEREDA, C., PRADA, M.A. y ACTIS, W. (2003). La Inserció Laboral de les persones amb discapacitats. Barcelona: Col-lecció Estudis Socials, Núm. 014, Colectivo loé. Fundació "La Caixa".

KÄMÄRÄINEN, P. (2002). Rethinking key qualificacions: towards a new framework. En KÄMÄRÄINEN, P., ATTWELL, G. y BROWN, A. (eds.), Transformation of learning in education and training: Key qualifications revisited (pp. 39-52). Luxembourg:

EUR-OP.

RODRIGUEZ MORENO, M.L. (1994). Educación para la carrera de personas con necesidades educativas especiales. $V$ Jornadas de la Asociación Catalana de Orientación Escolar y Profesional (ACOEP) sobre "Orientación Psicopedagógica y atención a la diversidad", Barcelona, 17 y 18 de marzo.

SHALOCK, R. (1999). Hacia una nueva concepción de la discapacidad. En M. VERDUGO y F. De BORJA JORDÁN (Coord.), Hacia una nueva concepción de la discapacidad. III Jornadas Científicas de Investigación sobre personas con discapacidad. Salamanca: Amarú.

SILVER, H. (1994). Exclusión social y solidaridad social: tres paradigmas. Revista Internacional del Trabajo, (5-6), 607.

Fecha de recepción: 10-06-2008

Fecha de revisión: 06-01-2009

Fecha de aceptación: 19-01-2010 


\section{ANEXO 1}

ENCUESTA PARA ALUMNADO QUE FINALIZA LA E.S.O.

Presentación: Un grupo de profesores de la Universidad de Córdoba estamos realizando un estudio sobre las opiniones de los estudiantes respecto a la Orientación Profesional y Vocacional recibida para enfocar vuestro futuro tras finalizar la ESO. Por ello, os agradecemos que contestéis a unas preguntas. Tanto vuestra identidad como las respuestas a estas preguntas serán tratadas de forma anónima, tal como establece la legislación al respecto.

Sexo: Mujer Hombre

Curso que estás realizando:

¿Has repetido curso? Sí
Año de nacimiento:

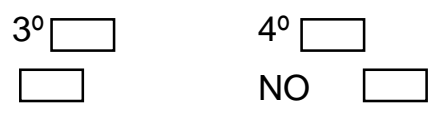

Sin contarte a ti, ¿cuántas personas viven habitualmente en tu casa?

Estudios del padre: $\quad$ Sin estudios

Primarios

Bachiller
Bachiller

Formación Profesional

Universidad

Estudios de la madre:

Sin estudios

Bachiller

Primarios

Formación Profesional

Bachiller

Universidad

Trabajo del padre:

Empleado $\square \quad$ Parado

Jubilado

Trabajo de la madre:

$\begin{array}{ll}\text { Empleada } & \square \\ \text { Parada } & \text { Sólo ama de casa } \\ \text { sí } \square & \text { Jubilada } \square\end{array}$

¿Tienes hermanos/as? Si

En caso afirmativo, titulación y ocupación de los hermanos/as.(repetir datos para cada hermano/a)

\begin{tabular}{|c|l|l|l|l|l|l|l|l|l|}
\hline $\begin{array}{c}N^{\circ} \text { de } \\
\text { hermanos }\end{array}$ & Sexo & Edad & $\begin{array}{c}\text { Sin } \\
\text { estudios }\end{array}$ & $\begin{array}{c}\text { Estudios } \\
\text { obligatorios }\end{array}$ & PGS & $\begin{array}{c}\text { Ciclo } \\
\text { Formativo }\end{array}$ & $\begin{array}{c}\text { Formación } \\
\text { Profesional }\end{array}$ & Universidad & $\begin{array}{c}\text { Ocupación que } \\
\text { desempeña }\end{array}$ \\
\hline $\begin{array}{c}1^{\circ} \\
\text { Hermano/a }\end{array}$ & & & & & & & & \\
\hline $\begin{array}{c}2^{\circ} \\
\text { Hermano/a }\end{array}$ & & & & & & & & \\
\hline $\begin{array}{c}3^{\circ} \\
\text { Hermano/a }\end{array}$ & & & & & & & & \\
\hline $\begin{array}{c}4^{\circ} \\
\text { Hermano/a }\end{array}$ & & & & & & & & \\
\hline $\begin{array}{c}5^{\circ} \\
\text { Hermano/a }\end{array}$ & & & & & & & & \\
\hline
\end{tabular}




\section{CUESTIONARIO}

4. ¿Qué tienes pensado hacer cuando acabes la ESO?

4.1. Seguir estudiando

4.2. Empezar a trabajar

4.3. Las dos cosas al mismo tiempo

4.4. No Sabe

5. Si vas a seguir estudiando, señala el tipo de estudios que piensas realizar cuando acabes la ESO.

5.1. Programas de Garantía Social (PGS)

5.2. Formación Profesional Ocupacional (FPO)

5.3. Ciclo Formativo

5.4. Bachillerato

5.5. No Sabe

6. Señala aquellos estudios de los que tienes información.(puedes señalar más de una opción)

6.1. Programas de Garantía Social (PGS)

6.2. Formación Profesional Ocupacional (FPO)

6.3. Ciclo Formativo

6.4. Bachillerato

6.5. No Sabe

7. Si vas a empezar a trabajar cuando acabes la ESO, ¿qué tipo de trabajo piensas realizar?

8. ¿Se han llevado a cabo actividades de Orientación Académica y Profesional durante este curso en tu Instituto?
a) sí
b) $\mathrm{NO}$

9. En caso afirmativo, ¿En qué momento del curso se ha llevado a cabo la Orientación?

9.1. A principio de curso

9.2. Hacia la mitad del curso

10. Las actividades de Orientación se han desarrollado en:

10.1. Un sólo día

10.2. Varios días

¿Cuántos?

11. En tu opinión, ¿el momento en que se ha impartido la Orientación ha sido el adecuado?
a) Sí
b) $\mathrm{NO}$

12. En tu opinión, ¿la duración que ha tenido la Orientación ha sido suficiente?
a) Sí
b) $\mathrm{NO}$

13. ¿Qué momento o momentos consideras que habrían sido el mejor o los mejores para realizar la Orientación?

13.1. A principio de curso

13.2. Hacia la mitad
A final del curso

En varios momentos 
14. ¿Qué duración consideras que deberían tener las actividades de Orientación?
a) Una vez a la semana
b) Una vez al mes
c) Una vez al trimestre
d) Una vez al año (durante varias jornadas seguidas)

15. ¿Crees que la Orientación debería empezar en los cursos anteriores a $3^{\circ}$ de la E.S.O.?
a) Sí
b) NO

16. ¿Sobre qué tipo de estudios se han centrado las actividades de Orientación en tu Instituto?(puedes señalar más de una opción)
a) Programas de Garantía Social (PGS)
c) Ciclos Formativos
b) Formación Profesional Ocupacional (FPO)
d) Bachillerato

17. ¿La Orientación recibida ha sido útil?
a) Mucho
c) Regular
e) Nada
b) Suficiente
d) Poco

18. ¿Has recibido Orientación en tu Instituto sobre Cursos de Formación Profesional Ocupacional (FPO)?(no nos referimos a los Ciclos de Formación o FP que se imparten en el instituto)
a) Sí
b) NO

19. En caso de haber recibido esta Orientación, ¿sobre qué tipo de cursos? (puedes señalar varias opciones)

19.1. Escuela Taller

19.2. Casas de Oficios

19.3. Experiencias Mixtas de Formación y Empleo

19.4. Otros

20. ¿Has recibido Orientación en tu Instituto sobre salidas laborales inmediatas?
a) Sí
b) $\mathrm{NO}$

21. En caso afirmativo, señala todas aquellas ocupaciones sobre las que te han orientado:

21.1. Actividades Agrarias

21.2. Actividades Físicas y Deportivas

21.3. Actividades Marítimo y Pesqueras

21.4. Administración

21.5. Artes Gráficas

21.6. Comercio y Marketing

21.7. Comunicación, Imagen y Sonido

21.8. Edificación y Obra Civil

21.9. Electricidad y Electrónica

21.10. Fabricación Mecánica

21.11. Hostelería y Turismo

21.12. Imagen Personal

21.13. Industria Alimentaria

21.14. Informática 
21.15. Madera y Mueble

21.16. Mantenimiento de vehículos autopropulsados

21.17. Mantenimiento y Servicios a la Producción

21.18. Química

21.19. Sanidad

21.20. Servicios Socioculturales y a la Comunidad

21.21. Textil, Confección y Piel

21.22. Vidrio y Cerámica

22. ¿Te han informado en tu Instituto sobre las instituciones a las que se puede acudir para la búsqueda de empleo?
a) Sí
b) NO $\square$

23. En caso afirmativo, señala aquellas instituciones sobre las que te han orientado (puedes señalar varias opciones).
a) Ayuntamiento
d) Asociación de Empresarios
b) Servicio Andaluz de Empleo
e) Diputación
c) Sindicatos
f) Otras

24. ¿Quién o quiénes te han dado la Orientación en tu Instituto? (puedes señalar varias opciones).

24.1. Orientador/a

24.2. Tutor/a

24.3. Profesor/a

24.4. Equipo Directivo

24.5. Otros

25. ¿Te han orientado personalmente en tu Instituto sobre qué tipo de salida escolar o profesional es la más adecuada para ti?
a) sí
b) $\mathrm{NO}$

26. En caso afirmativo,¿cuáles? (puedes señalar varias opciones)
a) Bachillerato
b) Ciclos Formativos
c) Programas de Garantía social
d) Formación Profesional Ocupacional
e) Ponerte a trabajar

27. ¿Estás de acuerdo con la salida escolar o profesional que te han propuesto en tu Instituto?
a) Sí
b) NO

28. ¿Has recibido Orientación sobre estudios o salidas laborales fuera de tu Instituto?

28.1. Familiares

28.2. Amigos/as y Vecinos/as

28.3. Monitores de Calle

28.4. Ayuntamiento

28.5. Diputación

28.6. Junta de Andalucía 
28.7. ONG

28.8. Internet

28.9. Otras vías

29. De todas las fuentes de Orientación a las que has tenido acceso, ¿cuál o cuáles de ellas consideras que han sido las más útiles?

29.1. Instituto

29.2. Familiares

29.3. Amigos/as y Vecinos/as

29.4. Monitores de Calle

29.5. Ayuntamiento

29.6. Diputación

29.7. Junta de Andalucía

29.8. ONG

29.9. Internet

29.10. Otras vías

30. ¿Qué comportamiento sueles tener durante el desarrollo de las clases?

30.1. Muy bueno

30.2. Bueno

30.3. Regular

30.4. Poco atento

31. ¿Crees que en tu Instituto existe una separación entre clases de "buenos/as estudiantes" y de "malos/as estudiantes"?
a) Sí
b) NO

32. En caso afirmativo, ¿qué criterios crees que tiene más en cuenta los profesores a la hora de agrupar a los alumnos y las alumnas en clases de "buenos/buenas estudiantes" o de "malos/malas estudiantes"?

32.1. Rendimiento Escolar

32.2. Comportamiento en clase

32.3. Otros

33. ¿Qué aspectos crees que deberían tener más en cuenta los Orientadores a la hora de aconsejar el camino que hay que seguir después de la ESO?

34. ¿Crees que, en este momento, tienes información suficiente para elegir lo que más te conviene?
34.1. Sí $\square$
34.2. NO

Muchas gracias por vuestra colaboración en la realización de esta investigación. 


\section{ANEXO 2}

ENTREVISTAS A LAS Y LOS ORIENTADORES: GUIÓN DE TRABAJO

\section{COMPOSICIÓN DEL DEPARTAMENTO DE ORIENTACIÓN}

1. Quién constituye el Departamento de Orientación en tu centro

2. Qué funciones desempeñas como miembro del Departamento de Orientación

3. Trayectoria profesional

\section{SATISFACCIÓN PERSONAL Y PROFESIONAL}

1. Valoración general de la función del orientador/a en relación.

- Con el alumnado

- Con el profesorado

- Con los padres/madres

- Con la administración

2. Nivel de satisfacción profesional

- Dificultades de la práctica orientadora

\section{3. ÁMBITOS DE ACTUACIÓN: ORIENTACIÓN ACADÉMICA-PROFESIONAL}

1. Perfil del alumnado

2. Tipología de las actuaciones

3. Tiempos y espacios dedicados a la orientación profesional

4. Planificación de la orientación profesional

5. Criterios para la orientación profesional al alumnado

6. Instrumentos y recursos en los procesos de orientación académica-profesional

7. Cooperación con entidades y profesionales de orientación de la zona

8. Experiencias y estrategias de intervención en los procesos de transición a la vida activa 